\title{
Transient eddy current flow metering
}

\author{
J Forbriger and F Stefani \\ Helmholtz-Zentrum Dresden - Rossendorf, PO Box 510119, D-01314 Dresden, \\ Germany \\ E-mail: F.Stefani@hzdr.de
}

\begin{abstract}
.
Measuring local velocities or entire flow rates in liquid metals or semiconductor melts is a notorious problem in many industrial applications, including metal casting and silicon crystal growth. We present a new variant of an old technique which relies on the continuous tracking of a flow-advected transient eddy current that is induced by a pulsed external magnetic field. This calibration-free method is validated by applying it to the velocity of a spinning disk made of aluminum. First tests at a rig with a flow of liquid GaInSn are also presented.
\end{abstract}

Keywords: flow measurement, inductive methods

Submitted to: Meas. Sci. Technol. 


\section{Introduction}

Compared to transparent fluids, liquid metals and semiconductor melts pose serious problems for the reliable determination of local velocities or integral flow characteristics [1]. Even the widely used ultrasonic Doppler velocimetry (UDV) faces difficulties when it comes to its application in very hot and/or chemically aggressive fluids, such as liquid steel or silicon. Fortunately, the high electrical conductivity that is responsible for the opaqueness of those fluids allows the utilization of magnetic inductive methods.

These methods bear on applying magnetic fields to the flowing fluid and measuring appropriate features, e.g. amplitudes, phases, or forces, of the flow induced magnetic fields. In the contactless inductive flow tomography (CIFT), entire three-dimensional flow fields are reconstructed from induced field amplitudes that are measured at many position around the fluid, to which one or a few external magnetic fields are applied [2, 3]. A recently developed flow rate sensor relies on the determination of magnetic phase shifts due to the flow [4, 12]. In the Lorentz force velocimetry (LFV) [5] one measures the force acting on a permanent magnet close to the flow, which results as a direct consequence of Newton's third law applied to the braking force acting by the magnet on the flow. With this technique, it is now possible to measure velocities of fluids with remarkably low conductivities, such as salt water [6].

A common drawback of all these methods is that the measured signal is not only dependent on the sought flow velocity, but also on the conductivity of the fluid. Usually, the signals are proportional to the so-called magnetic Reynolds number $R m=\mu_{0} \sigma V L$, where $\mu_{0}$ is the magnetic permeability constant, $\sigma$ the conductivity of the liquid, and $V$ and $L$ denote typical velocity and length scales of the relevant fluid volume. In most cases, the signal depends also on geometric factors, so that the measuring system has to be calibrated anyway. Further to this, the use of permanent magnets (for LVF) or of magnetic yoke materials (for the phase-shift method) set limitations to the ambient temperature at the position of the respective sensors.

The goal of the present paper is both to circumvent the necessity to calibrate the measurement system, and to mitigate the temperature limitation problem. The measurement system to be presented belongs to a wider class of time-of flight methods that utilize the existence of some traceable pattern in the fluid which is being advected by the flow. By measuring the time of flight of the advected pattern between two positions along the flow one can infer the flow velocity.

In one realization of this principle, the role of the pattern is played by some (unspecified) turbulence elements moving close to the wall of the fluid. If the flow direction is known, one can apply external magnetic fields at two positions and infer the flow speed from correlations of the magnetic signals induced by the turbulence elements that are passing by [7]. A similar correlation technique is presently under investigation in the time-of-flight LVF [8].

Another realization had already been developed in the 1960's by Zheigur and Sermons [9]. In their 'pulse method', the role of the traceable pattern is played by 
an eddy current system that is imprinted into the conducting medium by switching on or off the current in one excitation coil, and by registering the flow advected pattern by another coil situated downstream the flow. The instant at which the current in this detection coil crosses zero indicates the passing-by of the center of the eddy current system. The flow velocity is then determined by dividing the distance between the two coils by the measured time interval.

In [9], the method had been shown to allow to measure velocities of an aluminum bar from $20 \mathrm{~m} / \mathrm{s}$ down to $1 \mathrm{~m} / \mathrm{s}$, but not significantly lower. One reason for this limitation was the exclusive focus on identifying the instant of vanishing current in the detection coil by means of an oscillograph. For small velocities (or, more exactly, for small $R m$ ), when the decay of the eddy current is much faster then its advection, this instant smears out strongly so that its determination becomes less and less reliable.

In the present paper, we will apply a modern data acquisition system and appropriate analysis methods in order to infer the velocity from the complete data sets of the induced current in three detection coils, rather than only one particular instant in one single coil. With view on measuring the entire transient signal after switching, we dub this method as 'transient eddy current flow metering' (TEC-FM). After explaining the measuring principle and technical realization, we will apply it to the determination of the velocity of a rotating aluminum disk, and to the flow of GaInSn in a circular tube. The paper closes with some outlook for future improvements and for possible industrial application.

\section{Measuring system and principle}

The heart of our measuring system consists of three small detection coils embedded into one larger excitation coil (see figure 1). The excitation coil is installed parallel to the boundary of the fluid, so that the three detections coils are arranged in one row in direction of the flow.

The block wiring diagram is shown in figure 2. The signal for the excitation coil is produced by an AGILENT 33200A waveform generator and then amplified by a Rohrer PA2166 precision power amplifier. The induced signals measured by the three detection coils are amplified by FEMTO DLPVA-100-B-D amplifiers and then analyzed with a TECTRONIX DP07104 oscillograph.

In the following, we will focus on the case that the current in the excitation coil is on for $t<0$, and is being switched off at $t=0$ (note that the contrary case that the current is switched on at $t=0$ is not completely equivalent: the subtle differences will be discussed in the outlook). With our excitation technique we achieve a smooth and non-oscillatory decay with a typical fall time of approximately $100 \mu \mathrm{s}$. By this pulse, a ring-like eddy current system is induced in the nearby moving conductor. For a conducting half-space at rest, the evolution of this eddy current system can be given in a quasi-analytic form [10], which was also experimentally confirmed in a liquid metal experiment [11]. Here, we illustrate the ring-like eddy current system for the case of a 

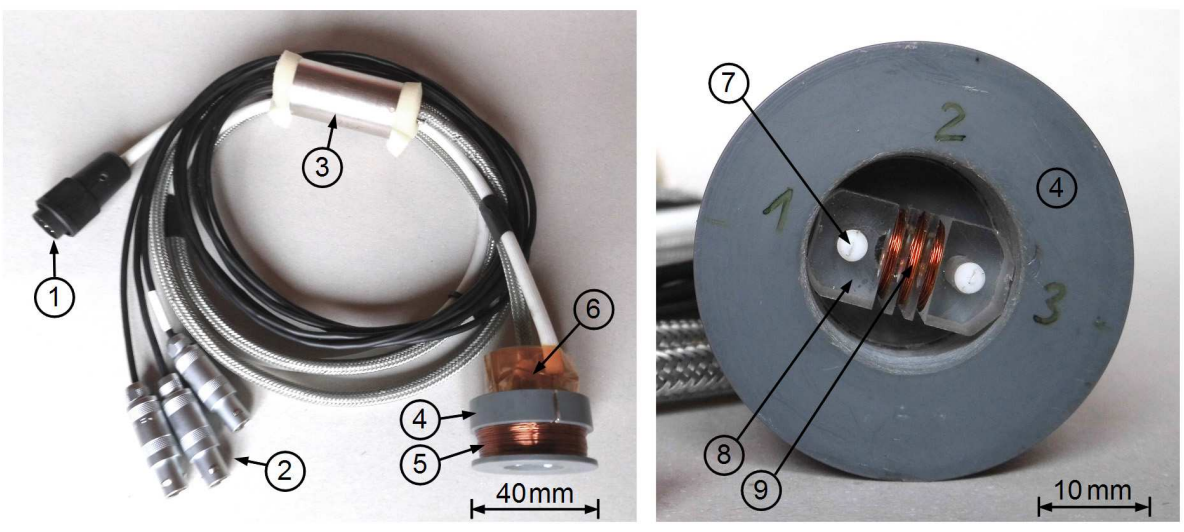

Figure 1. TEC-FM sensor with cables (left), and detailed view on the coil system (right). The plugin connector (1) connects the power supply to the excitation coil (5) consisting of 300 windings of copper wire with $0.5 \mathrm{~mm}$ diameter on the base body (4). The voltages induced in the three detection coils (9) are distributed in the adapter (3) to three connectors (2). The detection coils consist of 35 windings with diameter 0.1 $\mathrm{mm}$ that are wound on the winding former (8) that is fixed by screws (7) to the base body (4). The clamp (6) serves as a cable relief.

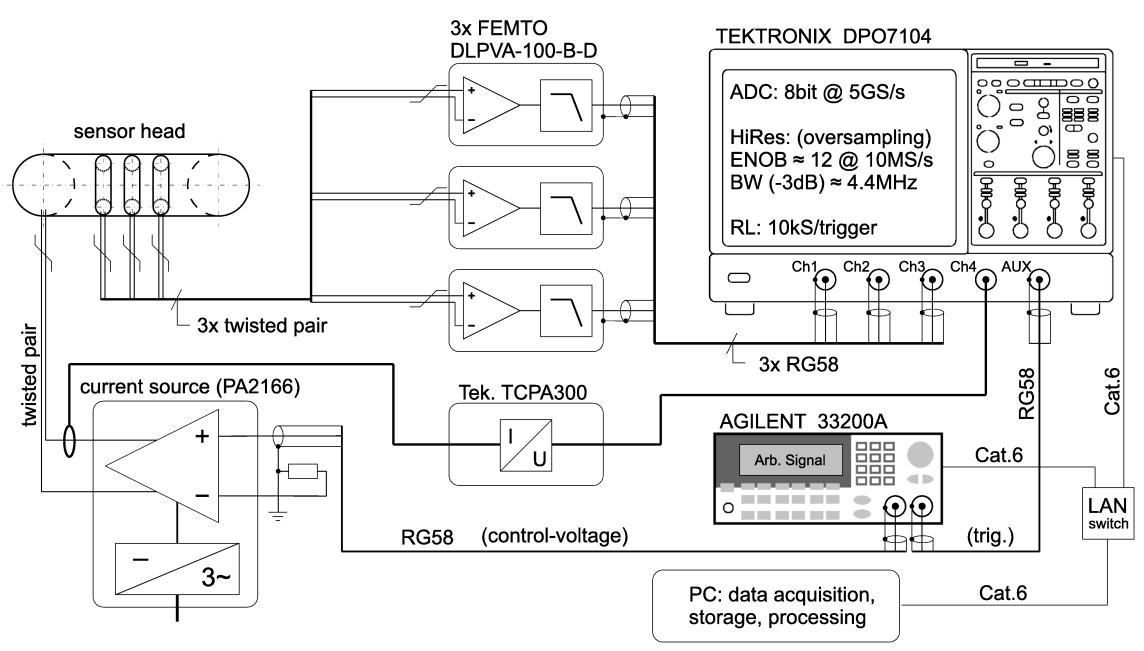

Figure 2. Block diagram of the measurement system.

solid electrical conductor moving to the right. Figure 3 shows the result of corresponding simulation with ANSYS multiphysics. The excitation coil is indicated below the body.

This eddy current, in turn, produces a magnetic field b outside the fluid, whose $b_{x}$-component (in flow direction) is zero exactly below the center of the (advected) eddy current ring. Close to this point, $b_{x}$ can be considered linear in $x$. If the detection coils are situated within this linear region, their signals can be exploited to identify the point of vanishing $b_{x}$ which represents the (moving) pole of the eddy current ring. The use of three coils enables to verify the linearity of the function $b_{x}(x)$. Yet, for the sake of simplicity, the following theoretical considerations will be restricted to the simpler two-coil system (figure 4). 


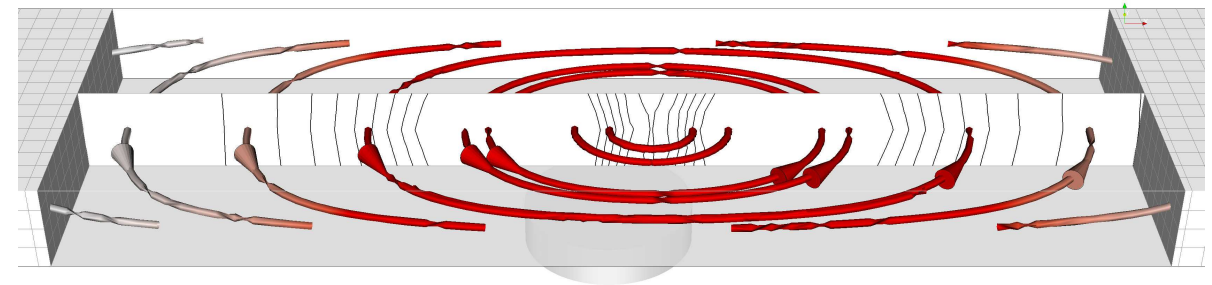

Figure 3. Simulated eddy current system induced in a right-moving solid body shortly after switching off the current in the emitter coil. The thick red arrows in the upper panel indicate the current lines, the thin gray lines are iso-contours of the current density.

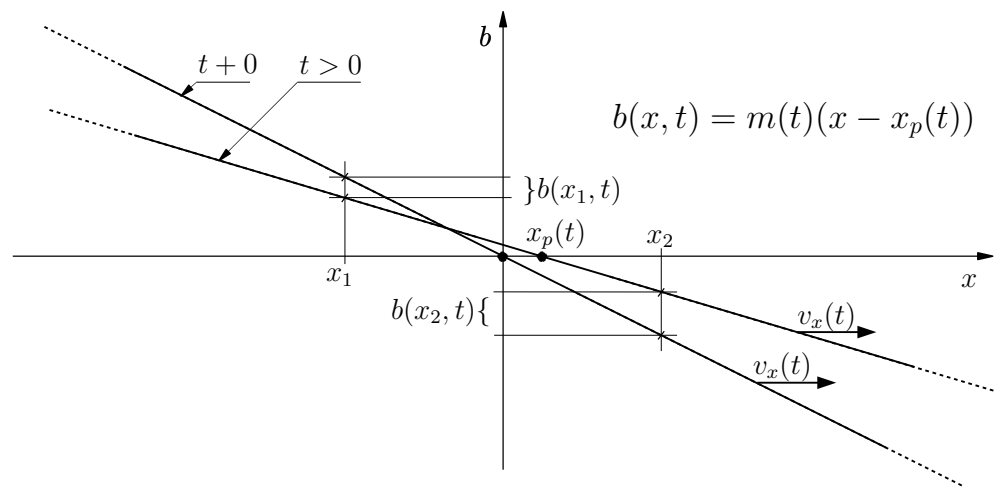

Figure 4. Schematic sketch of the evolution of the $b_{x}$ component of the magnetic field due to the eddy current.

Shortly after switching off the current in the excitation coil, the $b_{x}$ component along the flow direction $x$ can be parametrized in the following way (from here on, we replace $b_{x}$ by $\left.b\right)$ :

$$
b(x, t)=m(t)\left(x-x_{p}(t)\right)
$$

with the decay function

$$
m(t)=m_{0} \exp (-t / \tau)
$$

where $\tau$ is the typical decay time of the eddy current system in the liquid. $x_{p}(t)$ represents the time-dependent pole position of the flow-advected eddy current system which can, in turn, be interpreted as the time integral over the velocity of the fluid:

$$
x_{p}(t)=\int_{0}^{t} v_{x}(t) d t .
$$

Hence, the velocity of the pole of the eddy-current related magnetic field can be determined as

$$
v_{p}(t):=\frac{d x_{p}(t)}{d t} .
$$

The pole position $x_{p}$ at a given instant can be determined from the values $b\left(x_{1}, t\right)$ and 
$b\left(x_{2}, t\right)$ measured at the two detection coil positions $x_{1}$ and $x_{2}$, respectively:

$$
x_{p}(t)=x_{1}-\frac{b\left(x_{1}, t\right)\left(x_{2}-x_{1}\right)}{b\left(x_{2}, t\right)-b\left(x_{1}, t\right)} .
$$

This system of equations would be sufficient if the magnetic fields were indeed measured by Hall or Fluxgate sensors. However, since we are using pick-up coils (also with view on later high-temperature applications), our measured signal is actually the voltage in the detection coil which is proportional to the time derivative of the magnetic fields rather than the magnetic field itself.

The equation for this time-derivative of the magnetic field,

$$
\dot{b}(x, t)=\dot{m}(t)\left(x-x_{p}(t)\right)-m(t) \dot{x}_{p}(t)
$$

can be obtained from equation (1). The corresponding pole $x_{p p}$ of this time-derivative is derived from setting the r.h.s. of equation (6) to zero, and utilizing the relations $m(t) / \dot{m}(t)=\tau$, and $\dot{x}_{p}=v_{x}(t)$ :

$$
x_{p p}(t)=x_{p}(t)+\tau v_{x}(t) \text {. }
$$

The velocity of this pole of the magnetic field derivative results than as

$$
v_{p p}:=\dot{x}_{p p}(t)=v_{p}(t)+\tau \dot{v}_{p}(t) .
$$

We see that the velocity of the pole of the derivative $v_{p p}$ is identical to the velocity $v_{p}$ of the pole of the field itself as long as the latter is time-independent. In our method, we will trace indeed the pole of the time-derivative, which is for every instant given by

$$
x_{p p}(t)=x_{1}-\frac{\dot{b}\left(x_{1}, t\right)\left(x_{2}-x_{1}\right)}{\dot{b}\left(x_{2}, t\right)-\dot{b}\left(x_{1}, t\right)},
$$

quite in analogy with equation (5).

\section{Rotating aluminum disks}

In this section we will validate the TEC-FM method at a simple model for which the velocity is well known. For this purpose we chose a rotating aluminum disk of radius $165 \mathrm{~mm}$ and thickness $10 \mathrm{~mm}$, and apply our sensor at a radius of $145 \mathrm{~mm}$ (see figure 5]).

The velocity at this radius can be pre-adjusted by the disk rotation rate. Figure 6 shows the time evolution of the signals at the three receiver coils, for 11 chosen velocities between $0 \mathrm{~m} / \mathrm{s}$ and $5 \mathrm{~m} / \mathrm{s}$.

From these data we estimate the position of the pole (figure 7) for the first $2 \mathrm{~ms}$ after switching off the current. The black lines, drawn for comparison, would correspond to a perfect movement of the pole of the eddy current with the rotating disk.

We see that, at least during the first $1 \mathrm{~ms}$, the signal is very clean and allows to determine the speed of the pole. This is shown then in figure 8 , for which we have made an additional average over 10 pulsing events with an interval of $10 \mathrm{~ms}$ between them (so that we obtain one velocity value every $100 \mathrm{~ms}$ ). Obviously, the velocity can be recovered with high accuracy. 


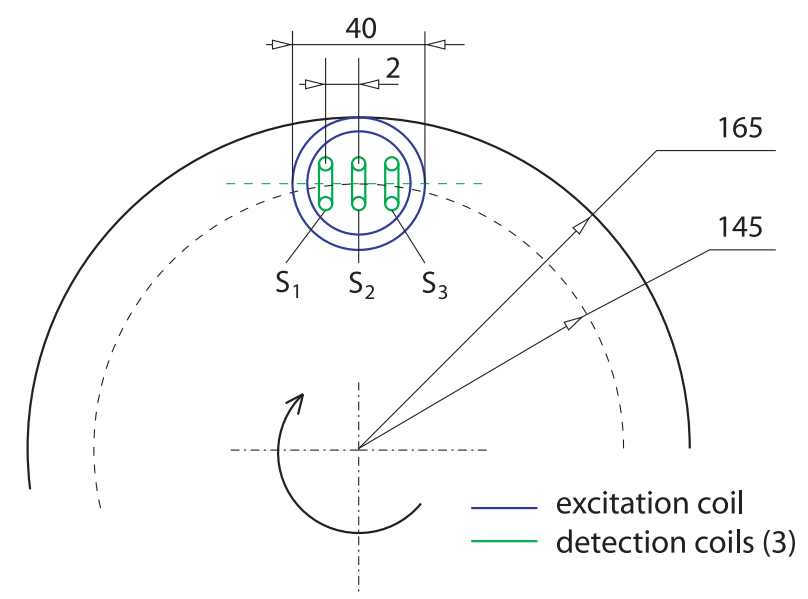

Figure 5. Velocity measurement for a rotating aluminum disk (not to scale).
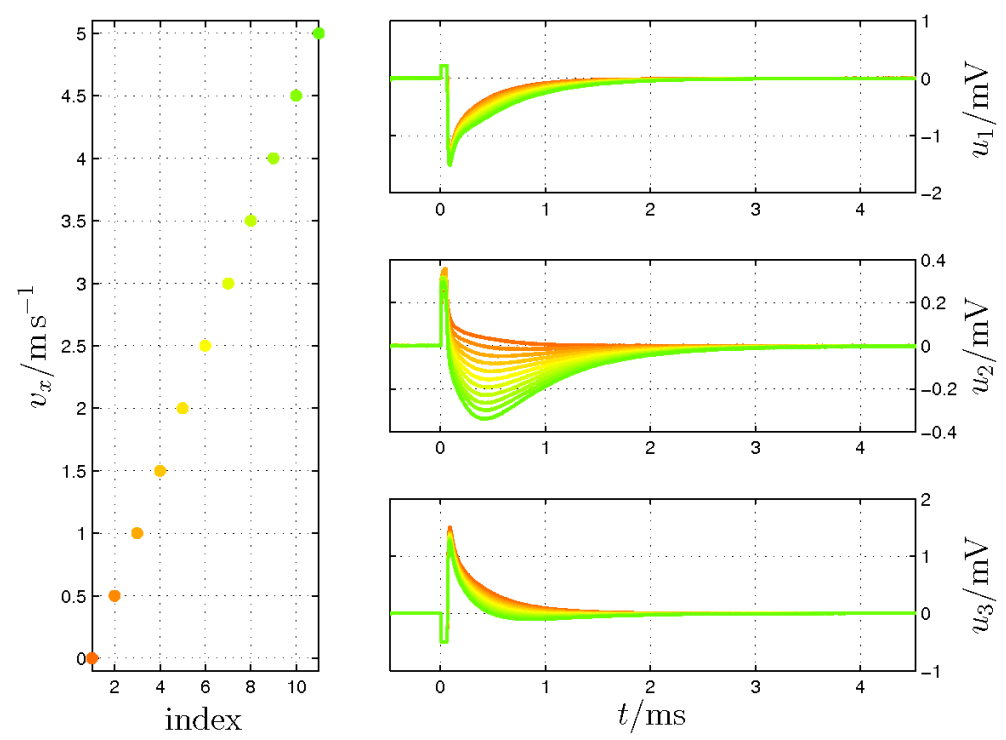

Figure 6. Time evolution of the voltages (raw signals) measured in the three receiver coils (right) for 11 pre-adjusted disk velocities $v_{x}$ (left).

\section{Flow of GaInSn in a pipe}

After having validated the method at a solid rotating disk, we apply it now to the case of a liquid metal flow in a pipe. We consider a flow of the eutectic alloy GaInSn (with the electrical conductivity $\sigma=3.3 \times 10^{6}$ ) in a circular plastic pipe of inner radius $27.3 \mathrm{~mm}$ (for more details of the test rig, see [12]). Figure 9 shows again the signal of the three coils for 15 different flow velocities (measured by an independent flow meter). Note that the magnetic field decay is much faster than in the aluminum case with its much higher conductivity, since the decay time is roughly $\tau=\mu_{0} \sigma d^{2}$, with $d$ a typical length scale of the order of the coil distance. This lower decay time would allow to provide velocity values approximately every $10 \mathrm{~ms}$.

Again, the time-dependence of the pole position $x_{p p}$ (figure 10) and the resulting 


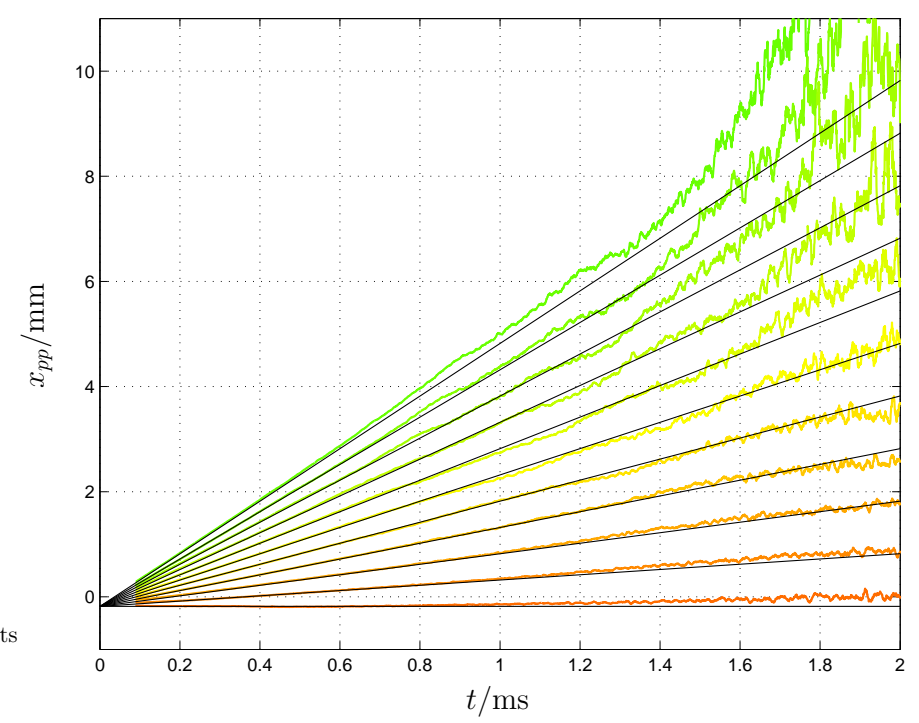

Figure 7. Time evolution of the reconstructed pole position $x_{p p}$ of the eddy current for the 11 pre-adjusted disk velocities $v_{x}$.

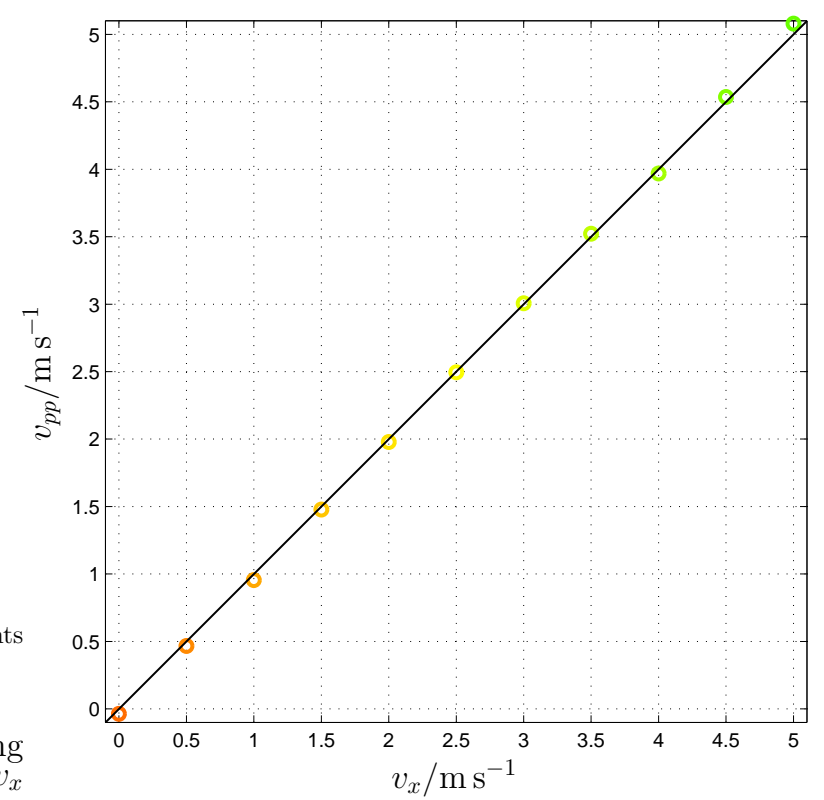

Figure 8. Inferred velocities $v_{p p}$ of the aluminum disk for the 11 pre-adjusted velocities $v_{x}$.

velocities $v_{p} p$ are shown (figure 11). Given the turbulent character of the pipe flow it is not surprising that the pole trajectory in figure 10 is much more noisy than the correponding pole trajectory for the aluminum disk. Consequently, we obtain also a larger deviation of the final velocity data (figure 11). Yet, the overall agreement with the pre-given velocity $v_{x}$ is quite reasonable, and might even be improved by a refined data analysis. 

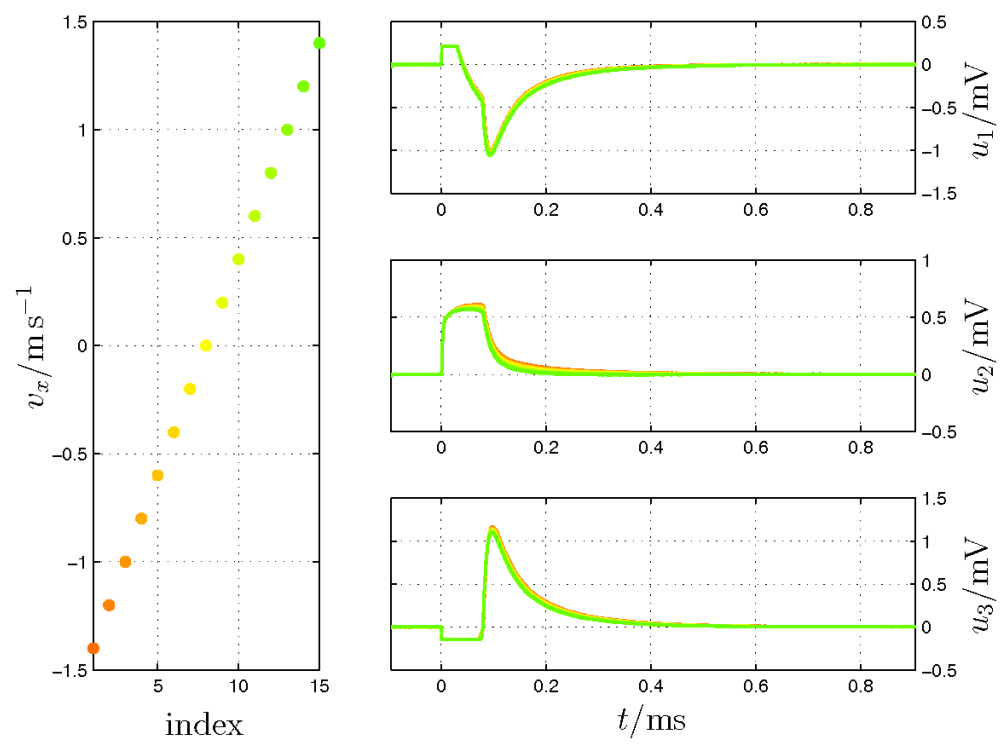

Figure 9. The same as Figure 6, but for the flow of GaInSn in a tube.

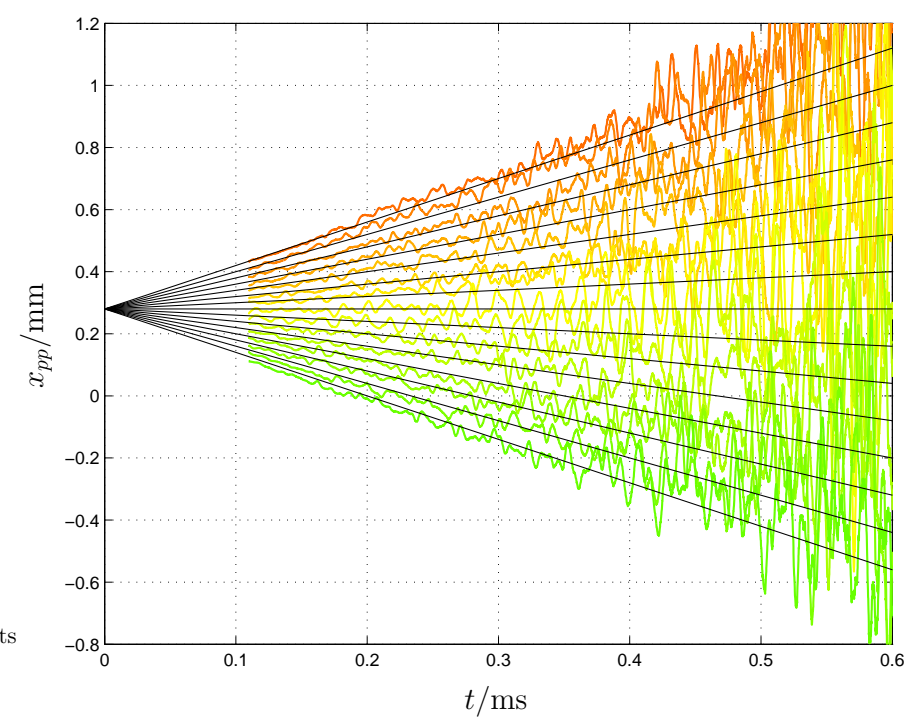

Figure 10. The same as Figure 7, but for the flow of GaInSn in a tube.

\section{Outlook}

We have modified, and significantly enhanced, the pulse method of Zheigur and Sermons [9] by using a compact design of three small detection coils embedded into a larger excitation coil, and by exploiting the complete signals measured at these coils for the determination of the flow velocity.

This 'transient eddy current flow-metering' (TEC-FM) has been validated at a rotating aluminum disk. First experiments at a flow of GaInSn show the applicability of the method for liquid metals.

The method is calibration-free since it does not depend on the conductivity of the moving body (at least, as long as the signals are still strong enough). A further 


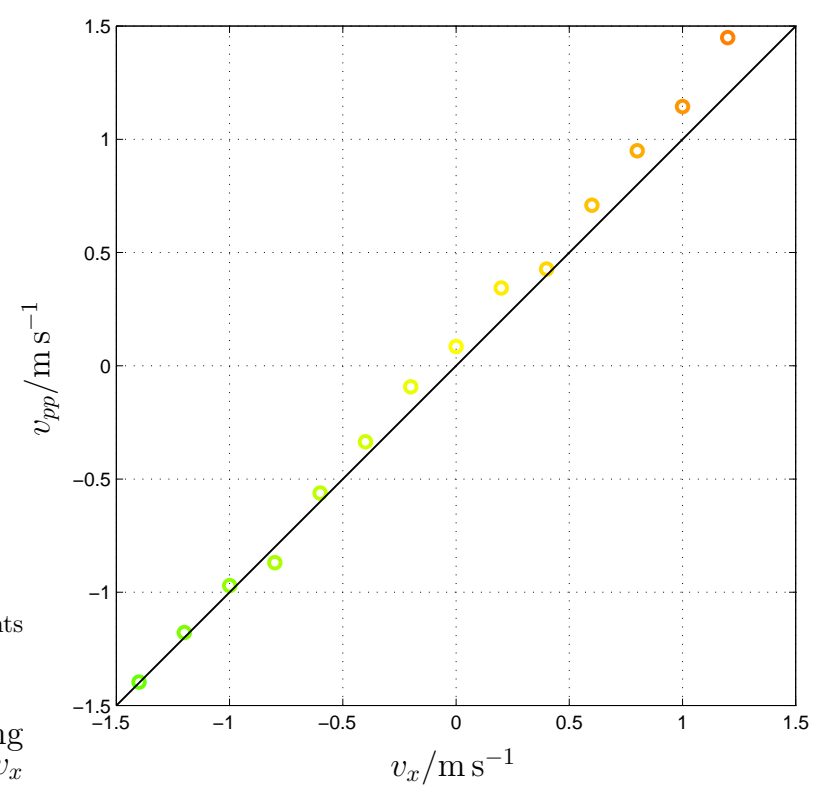

Figure 11. The same as Figure 8, but for the flow of GaInSn in a tube.

advantage of the sensor system is the avoidance of any magnetic material. Indeed, only air coils are used which can easily be adapted even to very hot environments such as for slab casters or within the pullers of Czochralski crystal growth.

A straightforward extension of the principle is to measure two dimensional flow structures close to the wall, just by adding two additional receiver coils orthogonal to the existing ones.

As mentioned above, there are subtle differences between switching on and switching off the current. These have to do with the $\mathbf{v} \times \mathbf{B}$ induction term which adds a weak $\infty$-shaped current system to the present ring-like one (as shown in figure 3 ). A detailed study of these differences, and of their potential to infer also the conductivity of the fluid, is left for future work.

\section{Acknowledgments}

This work was supported by Helmholtz-Gemeinschaft Deutscher Forschungszentren (HGF) in frame of the Helmholtz Alliance "Liquid metal technologies" (LIMTECH). We thank Dominique Buchenau, Hans Georg Krauthäuser and Jānis Priede for helpful discussions.

\section{References}

[1] Eckert S, Buchenau D, Gerbeth G, Stefani F and Weiss FP 2011 Some recent developments in the field of measuring techniques and instrumentation for liquid metal flows J. Nucl. Sci. Techn. 48 $490-9$

[2] Stefani F and Gerbeth G 2000 A contactless method for velocity reconstruction in electrically conducting fluids Meas. Sci. Techn. 11 758-65 
[3] Stefani F, Gundrum T and Gerbeth G 2004 Contactless inductive flow tomography Phys. Rev. E 70056306

[4] Priede J, Buchenau D and Gerbeth G 2011 Contactless electromagnetic phase-shift flowmeter for liquid metals Meas. Sci. Techn. 22055402

[5] Thess A, Votyakov E V and Kolesnikov Y 2006 Lorentz force velocimetry Phys. Rev. Lett. 96 164501

[6] Halbedel B et al 2014 A novel contactless flow rate measurement device for weakly conducting fluids based on Lorentz force velocimetry Flow Turb. Comb. 92 361-9

[7] Haubrich H and Julius E 1994 Flow meter US Patent US5426983A

[8] Dubovikova N, Karcher C and Kolesnikov Y 2014 Applications of Lorentz force techniques for flow rate control in liquid metals Proceedings of the 9th PAMIR International Conference on Fundamental and Applied MHD (Riga - Latvia, June 16-20, 2014) Vol 2 pp 118-122

[9] Zheigur BD and Sermons GY 1965 Pulse method of measuring the rate of flow of a conducting fluid Magnetohydrodynamics 1 (1) 101-104

[10] Tegopoulos J A and Kriezis E E 1985 Eddy Currents in Linear Conducting Media Elsevier: Amsterdam

[11] Forbriger J, Galindo V, Gerbeth G and Stefani F 2008 Measurement of the spatio-temporal distribution of harmonic and transient eddy currents in a liquid metal Meas. Sci. Technol. 19045704

[12] Priede J, Buchenau D and Gerbeth G 2009 Force-free and contactless sensor for electromagnetic flowrate measurements Magnetohydrodynamics 45 451-8 